\title{
Maladie hyperostosique et mode de vie : intérêt d'une démarche bio-archéologique. Exemple du cimetière du Couvent des Soeurs Grises de Beauvais (Oise), XVe-XVIIIe siècles
}

Diffuse idiopathic skeletal hyperostosis and way of life: a

bioarchaeologicalapproach. An example from the cemetery of the Convent of the

Soeurs Grises of Beauvais (Oise), XVth-XVIIIth centuries

\section{Sacha Kacki et Sébastien Villotte}

\section{(2) OpenEdition}

Journals

Édition électronique

URL : https://journals.openedition.org/bmsap/1315

DOI : $10.4000 /$ bmsap. 1315

ISSN : $1777-5469$

Éditeur

Société d'Anthropologie de Paris

Édition imprimée

Date de publication : 1 juin 2006

Pagination : 55-64

ISSN : 0037-8984

Référence électronique

Sacha Kacki et Sébastien Villotte, " Maladie hyperostosique et mode de vie : intérêt d'une démarche bio-archéologique. Exemple du cimetière du Couvent des Soeurs Grises de Beauvais (Oise), XVe-XVIIIe siècles ", Bulletins et mémoires de la Société d'Anthropologie de Paris [En ligne], 18 (1-2) | 2006, mis en ligne le 14 juin 2010, consulté le 01 juin 2021. URL : http://journals.openedition.org/bmsap/1315 ; DOI : https://doi.org/10.4000/bmsap.1315

Les contenus des Bulletins et mémoires de la Société d'Anthropologie de Paris sont mis à disposition selon les termes de la licence Creative Commons Attribution-NonCommercial-NoDerivatives 4.0 International License. 


\title{
MALADIE HYPEROSTOSIQUE ET MODE DE VIE : INTÉRÊT D'UNE DÉMARCHE BIO-ARCHÉOLOGIQUE. EXEMPLE DU CIMETIÈRE DU COUVENT DES SCEURS GRISES DE BEAUVAIS (OISE), XV ${ }^{\mathrm{e}}$-XVIII ${ }^{\mathrm{e}}$ SIÈCLES
}

\author{
DIFFUSE IDIOPATHIC SKELETAL HYPEROSTOSIS AND WAY OF LIFE: \\ A BIOARCHAEOLOGICAL APPROACH. \\ AN EXAMPLE FROM THE CEMETERY OF THE CONVENT OF THE SOEURS \\ GRISES OF BEAUVAIS (OISE), XVth-XVIIIth CENTURIES
}

\author{
Sacha KACKI, Sébastien VILLOTTE ${ }^{1}$
}

RÉSUMÉ

Dans le cadre d'études paléopathologiques, la maladie hyperostosique est parfois associée à des conditions de vie particulières, notamment à une diète trop riche. Les données médicales sur lesquelles se basent ces interprétations restent toutefois discutées. L'incidence de la maladie hyperostosique a été évaluée dans un échantillon de 305 individus adultes provenant d'un cimetière de couvent. Huit cas avérés et deux cas probables de la maladie ont été diagnostiqués. L'influence de l'âge a pu être vérifiée et une forte prédominance masculine est apparue. L'analyse des textes historiques ayant laissé supposer l'existence de lieux d'inhumation privilégiés, la répartition spatiale des malades a été étudiée. Elle indique une importante différence suivant les secteurs, tendant à accréditer la relation entre maladie hyperostosique et statut social des individus. Nos résultats appuient l'hypothèse d'une influence des conditions de vie sur l'expression de la maladie qui pourrait être imputable à une différence de comportements alimentaires ou de longévité des individus. Cette étude confirme l'intérêt de l'utilisation conjointe de données historiques, archéologiques, et paléopathologiques dans la reconstitution du mode de vie des populations du passé.

Mots-clés : maladie hyperostosique, paléopathologie, mode de vie, répartition spatiale.

\section{ABSTRACT}

Within the framework of paleopathological studies, diffuse idiopathic skeletal hyperostosis (DISH) is sometimes associated with a particular way of life, especially with an overly rich diet. Medical data upon which this interpretation is based are discussed. The prevalence of DISH was evaluated in a sample of 305 adults from a convent cemetery. Eight proven cases and two probable cases of the disease were diagnosed. The influence of age could be checked and a strong male prevalence appeared. As the analysis of the historical texts indicates the existence of privileged places of burial, the space distribution of cases was studied. It indicates an important difference according to sectors, supporting a relationship between DISH and social status of the individuals. Our results support the assumption of an influence of the way of life on the expression of the disease which could be ascribable to feeding behaviours or individual longevity differences. This study confirms the usefulness of the joint application of historical, archaeological, and paleopathological data in the reconstitution of the way of life of past populations.

Keywords: diffuse idiopathic skeletal hyperostosis, DISH, paleopathology, way of life, spatial distribution.

1. UMR 5199, PACEA, Laboratoire d'Anthropologie des Populations du Passé, Université Bordeaux 1, avenue des Facultés, 33405 Talence CEDEX, France, e-mail : sachakacki@hotmail.com 


\section{INTRODUCTION}

Décrite en détail pour la première fois par Forestier et Rotès-Querol (1950), la maladie hyperostosique (MH), nommée diffuse idiopathic skeletal hyperostosis (DISH) dans les pays anglophones, est une maladie du sujet âgé. Elle se caractérise par une ossification des tendons, ligaments et capsules articulaires au niveau de leurs insertions osseuses, appelées enthèses (Morlock 1992). La manifestation la mieux connue de la maladie est une coulée osseuse au niveau du rachis, pouvant produire l'ankylose d'un nombre variable de vertèbres. Cette coulée s'observe généralement au niveau des faces antérieure et latérale droite du rachis thoracique (Forestier, Rotès-Querol 1950 ; Resnick 2002). Les enthèses extra-rachidiennes peuvent également être concernées par des productions osseuses exubérantes (Resnick et al. 1975 ; Resnick 2002).

Dans le cadre d'études paléopathologiques, la MH a parfois été associée à des conditions de vie particulières, notamment à une diète trop riche (Rogers, Waldron 2001 ; Jankauskas 2003). Cette hypothèse est fondée sur les données médicales faisant état d'une relation entre la MH et l'obésité, le diabète ou la goutte (Julkunen et al. 1971 ; Utsinger 1985 ; Lussier, de Medicis 1991). Cependant, l'étiologie de la maladie restant encore inconnue (Resnick 2002), il apparaît prématuré de généraliser un lien entre MH et mode de vie au sein d'une population archéologique. L'utilisation conjointe, dans la présente étude, de données historiques (statut social des inhumés), archéologiques (sectorisation des tombes), et paléopathologiques contribue à cette discussion.

\section{MATÉRIEL ET MÉTHODES}

\section{Échantillon}

Le matériel ostéologique étudié provient du cimetière du couvent des Sœurs Grises, situé à Beauvais (Oise, France). La fouille quasi-exhaustive du site, réalisée en 1992, a permis la mise au jour de plus de 350 inhumations et d'une cinquantaine d'ossuaires et de réductions de corps (Fémolant 1992). Les squelettes étaient bien conservés et n'avaient, en général, subi aucune perturbation physique ou chimique macroscopiquement visible (Chevalier 1993). L'état de conservation des ossements semble, de plus, avoir été homogène entre les différentes parties du cimetière (Chevalier, communication personnelle). L'échantillon étudié se compose des restes osseux de 305 individus adultes, provenant de quatre ensembles archéologiques distincts :

- le cimetière ouvert : 214 individus ;

- le Chøur des Dames : 25 individus ;

- la chapelle : 43 individus;

- la chapelle cémétériale : 23 individus.

La détermination du sexe a été réalisée à partir de mesures coxales (méthode probabiliste : Murail et al. 2005) et de mesures extra-coxales (diagnose sexuelle secondaire : Murail et al. 1999). Pour chacune de ces méthodes, une probabilité de 0,95 a été requise pour l'attribution d'un sexe. La répartition sexuelle des individus $(t a b l . I)$ indique une prédominance des sujets féminins dans la population. Des hommes sont toutefois présents dans les différentes zones d'inhumation, et en proportion plus importante dans la chapelle.

\begin{tabular}{lccccccc} 
& \multicolumn{2}{c}{ Masculin } & \multicolumn{2}{c}{ Féminin } & \multicolumn{2}{c}{ Indéterminé } & \multirow{2}{*}{ Total } \\
& $\mathrm{N}$ & $\%$ & $\mathrm{~N}$ & $\%$ & $\mathrm{~N}$ & $\%$ & $\mathrm{~N}$ \\
\hline \hline Cimetière ouvert & 17 & 7,9 & 142 & 66,4 & 55 & 25,7 & 214 \\
\hline Chœur des Dames & 3 & 12,0 & 18 & 72,0 & 4 & 16,0 & 25 \\
\hline Chapelle & 11 & 25,6 & 26 & 60,5 & 6 & 13,9 & 43 \\
\hline Chapelle cémétériale & 1 & 4,4 & 16 & 69,6 & 6 & 26,0 & 23 \\
\hline Toutes zones & 32 & 10,5 & 202 & 66,2 & 71 & 23,3 & 305 \\
\hline \hline
\end{tabular}

Tabl. I - Répartition sexuelle des individus suivant les zones d'inhumation. Table I-Distribution by sex of the individuals according to burial area. 
L'estimation de l'âge au décès a fait appel à deux indicateurs :

- remodelage de la surface sacro-pelvienne iliaque (Schmitt 2005) ;

- fusion de la crête iliaque à l'ilium et fusion de l'extrémité sternale de la clavicule (Owings-Webb, Suchey 1985).
Afin de faciliter la lisibilité des résultats, seules deux classes d'âge ont été retenues dans cette étude : les moins de cinquante ans et les plus de cinquante ans (tabl. II).

\begin{tabular}{|c|c|c|c|c|c|c|c|c|c|c|c|c|}
\hline \multirow[b]{2}{*}{ Classe d'âge } & \multicolumn{3}{|c|}{ Hommes } & \multicolumn{3}{|c|}{ Femmes } & \multicolumn{3}{|c|}{ Indéterminés } & \multicolumn{3}{|c|}{ Total } \\
\hline & $<50$ ans & $>50$ ans & I & $<50$ ans & $>50$ ans & I & $<50$ ans & $>50$ ans & I & $<50$ ans & $>50$ ans & $I$ \\
\hline Cimetière ouvert & 5 & 2 & 10 & 62 & 22 & 58 & 4 & 5 & 46 & 71 & 27 & 114 \\
\hline Chœur des Dames & 0 & 1 & 2 & 8 & 4 & 6 & 1 & 1 & 2 & 9 & 6 & 10 \\
\hline Chapelle & 2 & 3 & 6 & 9 & 3 & 14 & 1 & 1 & 4 & 12 & 9 & 24 \\
\hline Chapelle cémétériale & 0 & 0 & 1 & 6 & 3 & 7 & 2 & 0 & 4 & 8 & 3 & 12 \\
\hline Toutes zones & 7 & 6 & 19 & 85 & 32 & 85 & 8 & 7 & 56 & 100 & 45 & 160 \\
\hline
\end{tabular}

Tabl. II - Répartition par âge au décès des individus suivant le sexe et la zone d'inhumation (I = âge indéterminé). Table II-Age at death distribution according to sex and burial area $(I=$ unspecified age $)$.

\section{Données historiques (d'après Chevalier 1993)}

Le couvent a été occupé par la communauté religieuse entre 1480 et la fin du XVIII ${ }^{\mathrm{e}}$ s. Le premier devoir des Sœurs Grises était de soigner les malades, quels qu'ils soient. À partir de 1630, le nouvel axe de service des sœurs fut l'éducation des enfants que leur confiaient les familles de Beauvais. En 1502, la communauté obtint pour le couvent le droit d'inhumer. Le cimetière reçu ses premières reliques le 20 août 1505 .

Ces données laissent supposer trois origines possibles pour les inhumés : des membres de la communauté religieuse ${ }^{2}$, des malades ou des enfants pris en charge par les sœurs, et enfin, des habitants de la ville. Les sources textuelles font état de plusieurs cas où un notable sollicitait d'être enterré dans un lieu particulier. Ainsi, les bienfaiteurs du couvent, bourgeois ou religieux, désiraient volontiers être inhumés dans la chapelle ou la chapelle cémétériale. Entre autres exemples, Jehan de la Brosse, curé de la Paroisse Saint-Laurent, demanda le 3 septembre 1644 à être inhumé « au lieu d'estime, proche

2. Outre les sœurs, plusieurs hommes faisaient partie de la communauté : un supérieur, un chapelain, un confesseur et un père temporel, gérant les ressources du couvent. le grand autel ». De même, le chanoine Besgne, docteur en théologie, émit le souhait d'être inhumé dans la chapelle, près de son oncle et dans une tombe semblable.

Ceci nous a conduit à définir deux groupes d'inhumés selon leur localisation spatiale. Ainsi, les individus provenant du cimetière ouvert et du Chœur des Dames, zones d'inhumation n'ayant pas fait l'objet, à notre connaissance, de demandes particulières, seront distingués dans cette étude de ceux provenant des deux chapelles.

\section{Diagnostic de la maladie hyperostosique}

Le diagnostic de la $\mathrm{MH}$ a été réalisé d'après les critères d'Utsinger (1985). Les cas avérés de la maladie sont caractérisés par une coulée osseuse antéro-latérale, sans distinction de côté, au niveau d'au moins quatre vertèbres adjacentes. Les cas probables sont définis par l'association d'une coulée osseuse antéro-latérale au niveau d'au moins deux vertèbres adjacentes et d'enthésopathies périphériques symétriques au niveau de l'insertion du triceps brachial sur l'ulna, du quadriceps fémoral sur la patella ou du triceps sural sur le calcanéus. Les critères d'exclusion sont une réduction anormale de l'espace intervertébral et/ou une ankylose vertébrale postérieure. 
Remarques :

- Resnick (2002) note que, fréquemment, les atteintes rachidiennes considérées comme des ankyloses sont en fait des excroissances osseuses interdigitées. En conséquence, les coulées osseuses rachidiennes continues (fig. 1) et discontinues (fig. 2) ont été prises en compte dans cette étude. Ces ossifications se développent à partir du corps vertébral, contrairement aux ostéophytes d'arthrose (Lagier 1987 ; Van de Berg et al. 2003), et passent obliquement à distance des disques intervertébraux, contrairement aux syndesmophytes rencontrés dans les spondylarthropathies (Crubézy 1990 ; Van de Berg et al. 2003).

- Pour les atteintes du squelette appendiculaire, une longueur minimum de $2 \mathrm{~mm}$ a été requise pour considérer les enthésophytes (modifié d'après Crubézy 1990).
L'enthèse constituant également l'une des cibles privilégiées des maladies appartenant au groupe des spondylarthropathies (pour revue voir Rogers et Waldron 1995, Benjamin et McGonagle 2001), les enthésopathies périphériques n'ont été prises en compte comme critère diagnostic de la MH qu'en l'absence, chez les individus, de signes évocateurs d'une spondylarthropathie : en particulier une sacro-iliite, des atteintes érosives au niveau des os des pieds ou des mains, des manifestations inflammatoires au niveau du rachis.

- Les cas possibles de la maladie n'ont pas été pris en compte dans cette étude, les remodelages périphériques considérés pouvant être, selon nous, la simple conséquence d'un processus dégénératif lié à la sénescence.

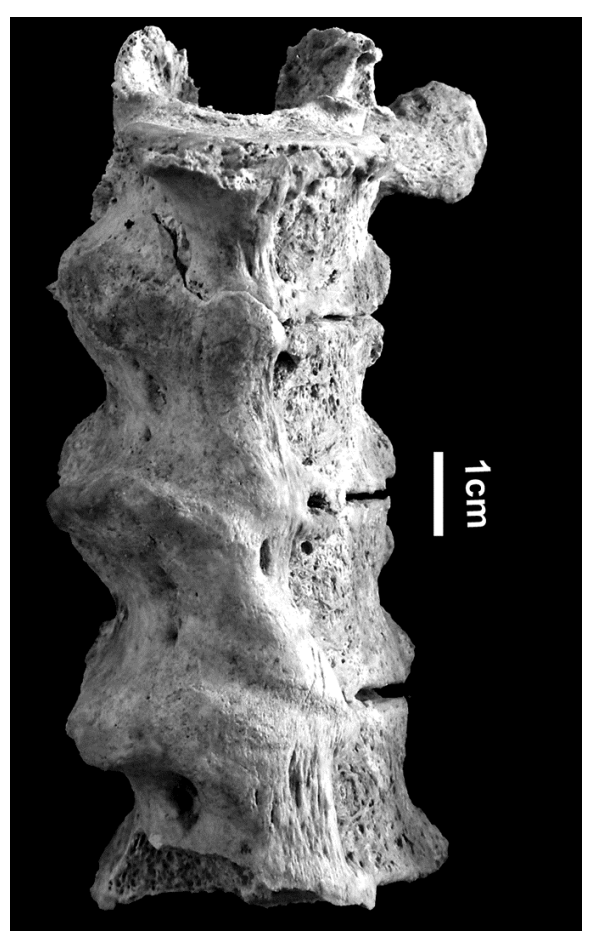

Fig 1 - Coulée osseuse antéro-latérale droite continue au niveau du rachis thoracique.

Fig. 1-Continuous new bone formation on right-hand side in the thoracic region.

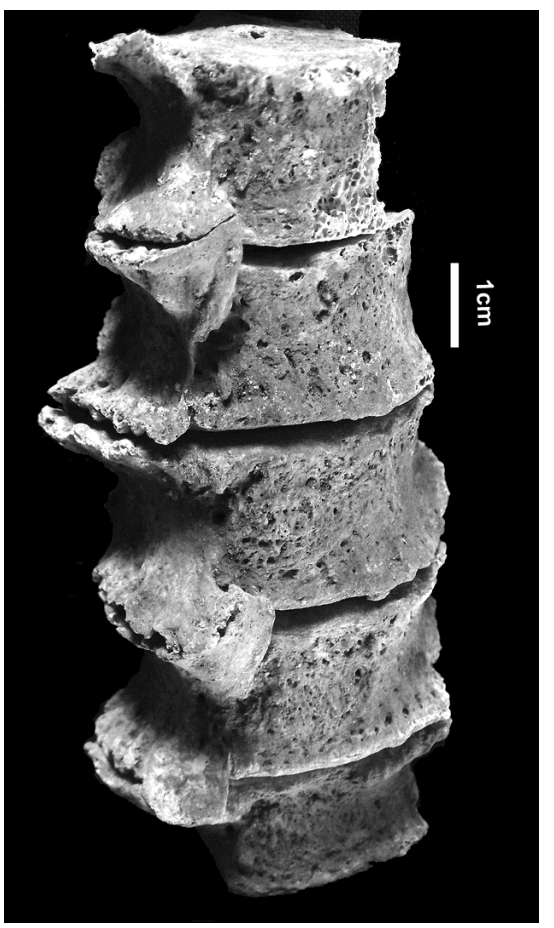

Fig. 2 - Coulée osseuse antéro-latérale droite discontinue au niveau du rachis thoracique.

Fig. 2-Discontinuous new bone formation on right-hand side in the thoracic region. 


\section{Fréquences et méthodes statistiques}

Suivant les recommandations de Rogers et Waldron (1995), les fréquences ont été calculées par le rapport entre le nombre d'individus atteints de $\mathrm{MH}$ et l'effectif total. Deux fréquences seront données, la première ne tenant compte que des cas avérés de la maladie, la seconde correspondant aux cas avérés et probables.

Le traitement statistique des résultats a été réalisé à l'aide du logiciel Statistica 7. Le test exact de Fisher et le test de comparaison des proportions (CP) ont été utilisés. Le seuil de signification de 0,05 a été requis.

\section{RÉSULTATS}

\section{Fréquence générale dans la population}

L'examen des restes osseux des 305 individus adultes a permis de diagnostiquer huit cas avérés et deux cas probables de MH (tabl. III).

\begin{tabular}{|c|c|c|c|c|}
\hline Zone & Individu & Sexe & $\hat{A} g e$ & Diagnostic \\
\hline \multirow{5}{*}{ Cimetière ouvert } & 3003 & F & Indéterminé & Probable \\
\hline & 3004 & $\mathrm{~F}$ & Indéterminé & Avéré \\
\hline & 3129 & $\mathrm{~F}$ & $>50$ ans & Avéré \\
\hline & 3137 & $\mathrm{~F}$ & Indéterminé & Avéré \\
\hline & 3210 & $M$ & $<50$ ans & Avéré \\
\hline \multirow{4}{*}{ Église } & 206 & $\mathrm{M}$ & $>50$ ans & Avéré \\
\hline & 215 & M & Indéterminé & Probable \\
\hline & 258 & $\mathrm{M}$ & Indéterminé & Avéré \\
\hline & 261 & $\mathrm{M}$ & $>50$ ans & Avéré \\
\hline Chapelle cémétériale & 451 & M & Indéterminé & Avéré \\
\hline
\end{tabular}

Tabl. III - Cas avérés et probables de MH selon la zone d'inhumation. Table III-Proven and probable cases of DISH according to burial area.

Chez tous les individus, la coulée osseuse est située au niveau de la partie antéro-latérale droite du rachis thoracique, s'étendant parfois au rachis lombaire. Le bon état de conservation et de représentation des restes osseux a permis d'écarter le diagnostic différentiel de spondylarthropathies ou la coexistence de ces maladies et de la $\mathrm{MH}$, aucun des individus ne présentant de sacroiliite ou d'atteintes érosives des os des mains ou des pieds.
Seuls deux squelettes (sépultures 3003 et 451) sont partiellement représentés. Toutefois, pour ces deux individus, l'aspect caractéristique en « coulée de bougie » nous est apparu suffisant pour établir le diagnostic.

La fréquence de la pathologie dans la population (2,6 \%-3,3\%) est relativement faible comparée à celles rencontrées dans les populations actuelles (tabl. IV) et anciennes (tabl. V).

\begin{tabular}{lcccc}
\hline \hline \multirow{2}{*}{ Origine } & \multicolumn{2}{c}{ Effectif } & \multicolumn{2}{c}{ Fréquence MH (\%) } \\
& Hommes & Femmes & Hommes & Femmes \\
\hline \hline Finlande & 4225 & 4768 & 3,8 & 2,6 \\
\hline Japon & 989 & 1872 & 7,5 & 0,6 \\
\hline Jérusalem & 624 & 612 & 22,4 & 13,4 \\
\hline Afrique du Sud & 408 & 1092 & 3,8 & 4,2 \\
\hline \hline
\end{tabular}

Tabl. IV - Fréquence de la MH chez les adultes de plus de 40 ans dans des populations actuelles, selon le sexe des individus (in Morlock 1992).

Table IV-Frequency of DISH in present populations for adults over 40 years according to gender (in Morlock 1992). 


\begin{tabular}{|c|c|c|c|c|c|c|c|}
\hline \multirow[t]{2}{*}{ Époque } & \multirow[t]{2}{*}{ Origine } & \multirow[t]{2}{*}{ Effectif } & \multicolumn{2}{|c|}{ MH avérée } & \multicolumn{2}{|c|}{$\begin{array}{c}\text { MH avérée ou } \\
\text { probable }\end{array}$} & \multirow[t]{2}{*}{ Auteur } \\
\hline & & & $\mathrm{N}$ & $\%$ & $\mathrm{~N}$ & $\%$ & \\
\hline Moderne & Coimbra (Portugal) & 51 & 14 & 27,4 & 19 & 33,4 & \multirow{2}{*}{ Cunha 1993} \\
\hline \multirow{2}{*}{ Moyen Âge } & Coimbra (Portugal) & 44 & 5 & 11,4 & 5 & 11,4 & \\
\hline & Canac (Aveyron) & 32 & 2 & 6,3 & 5 & 15,6 & Crubézy et al. 1998 \\
\hline \multirow[b]{2}{*}{ Haut Moyen Âge } & Raucourt (Lorraine) & 27 & 1 & 3,7 & 2 & 7,4 & \multirow[b]{2}{*}{ Vidal 2000} \\
\hline & $\begin{array}{l}\text { Chatel St Germain } \\
\text { (Lorraine) }\end{array}$ & 35 & 1 & 2,8 & 2 & 5,6 & \\
\hline
\end{tabular}

Tabl. V - Fréquence de la MH dans différentes populations anciennes (critères diagnostiques différents selon les auteurs). Table V-Frequency of DISH in various past populations (diagnostic criteria differ between authors).

\section{Répartition des malades par classe d'âge}

Une classe d'âge a pu être déterminée pour quatre des 10 individus atteints de $\mathrm{MH}$ (tabl. III). Un seul individu de moins de 50 ans est concerné $(1,0 \%)$ alors que $6,7 \%$ des individus âgés $(\mathrm{N}=3)$ présentent les signes caractéristiques de la maladie. Cette augmentation de la fréquence de la $\mathrm{MH}$ avec l'âge concorde avec les données de la littérature médicale (Morlock 1992 ; Resnick 2002).

\section{Répartition des malades par sexe}

Parmi les 202 femmes identifiées sur le site, seules trois présentent les marques indiscutables de la maladie et une ses signes probables. La fréquence de la $\mathrm{MH}$ dans la population féminine $(1,5 \%-2 \%)$ est relativement faible (tabl. IV). Six cas masculins, se répartissant en cinq cas avérés et un cas probable, ont été diagnostiqués pour un effectif total de 32 individus. La fréquence de la maladie chez les hommes (15,6\%-18,8 \%) est nettement supérieure à la fréquence féminine (Fisher ; $\mathrm{p}=0,0015$ - Fisher ; p = 0,0006), en accord avec la plupart des données épidémiologiques (tabl. IV).

\section{Répartition spatiale des malades}

\section{Le cimetière ouvert et le Chour des Dames}

Toutes les femmes atteintes de MH proviennent du cimetière ouvert. La fréquence féminine de la maladie y reste cependant relativement faible $(2,1 \%-2,8 \%)$. Un seul homme de cette zone (5,9\% de l'effectif masculin) présente les signes de la maladie. Aucun cas de MH n'a été diagnostiqué dans le Chøur des Dames. La fréquence totale dans ces deux zones est de $5 \%$ chez les hommes et $1,9 \%-2,5 \%$ chez les femmes.

\section{La chapelle et la chapelle cémétériale}

Trois cas avérés et un cas probable de MH ont été diagnostiqués chez les hommes inhumés dans la chapelle (27,3\%-36,4\%). Le seul homme identifié dans la chapelle cémétériale est également atteint. La fréquence d'hommes malades au sein de ce sous-ensemble archéologique est de 33,3\%-41,7\%. Cette fréquence hors norme dans la population masculine contraste avec l'absence de $\mathrm{MH}$ chez les individus féminins de ces zones.

\section{Comparaison entre les secteurs d'inhumation (tabl. VI)}

Dans notre échantillon, il apparaît une forte hétérogénéité entre la fréquence des malades dans le cimetière ouvert et le Chœur des Dames (secteur 1) et celle observée dans les chapelles (secteur 2). Cette différence reste toutefois non significative (Fisher ; $p=0,085-$ Fisher $; p=0,054)$. La proportion de femmes atteintes de la $\mathrm{MH}$ est relativement faible dans les deux secteurs, et la maladie ne présente pas de distribution particulière $(\mathrm{CP} ; \mathrm{p}=0,356-\mathrm{CP} ; \mathrm{p}=0,257) . \mathrm{Au}$ contraire, la proportion d'hommes atteints dans la chapelle et la chapelle cémétériale diffère significativement de celle observée dans le reste du cimetière $(\mathrm{CP} ; \mathrm{p}=0,039-\mathrm{CP} ; \mathrm{p}=0,014)$. La distribution par âge au décès des individus masculins ne variant pas de manière significative entre les deux secteurs (Fisher ; $\mathrm{p}=0,59$ ), cette différence laisse supposer une répartition spatiale non aléatoire des malades au sein du cimetière (fig. 3). 


\begin{tabular}{|c|c|c|c|c|c|c|}
\hline & \multirow[t]{2}{*}{ Localisation } & \multirow[t]{2}{*}{ Effectif } & \multicolumn{2}{|c|}{ Cas avérés } & \multicolumn{2}{|c|}{$\begin{array}{l}\text { Cas avérés } \\
+ \text { probables }\end{array}$} \\
\hline & & & $\mathrm{N}$ & $\%$ & $\mathrm{~N}$ & $\%$ \\
\hline \multirow{2}{*}{ Femmes et Hommes } & Secteur 1 & 180 & 4 & 2,2 & 5 & 2,8 \\
\hline & Secteur 2 & 54 & 4 & 7,4 & 5 & 9,3 \\
\hline \multirow{2}{*}{ Femmes } & Secteur 1 & 160 & 3 & 1,9 & 4 & 2,5 \\
\hline & Secteur 2 & 42 & 0 & 0,0 & 0 & 0,0 \\
\hline \multirow{2}{*}{ Hommes } & Secteur 1 & 20 & 1 & 5,0 & 1 & 5,0 \\
\hline & Secteur 2 & 12 & 4 & 33,3 & 5 & 41,7 \\
\hline
\end{tabular}

Tabl. VI - Effectif et fréquence des cas de MH selon la localisation.

Table VI-Number and frequency of cases of DISH according to burial location.

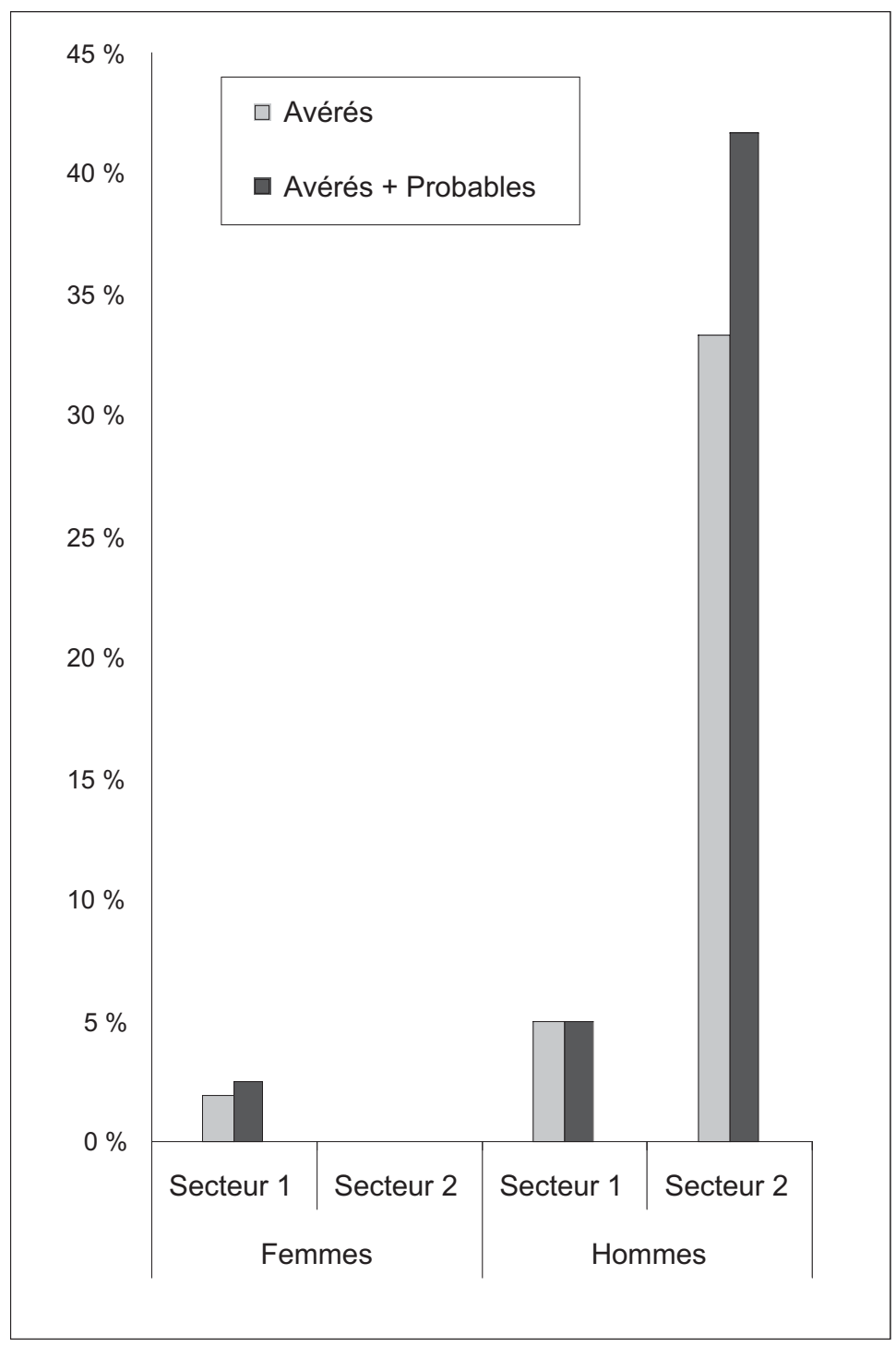

Fig. 3 - Fréquence de la MH entre secteurs d'inhumation, selon le sexe des individus.

Fig. 3-Frequency of DISH between burial sectors, according to sex. 


\section{DISCUSSION}

La fréquence de la $\mathrm{MH}$ dans l'échantillon étudié (2,6\%-3,3\%) est relativement faible au regard de celles rencontrées dans les populations actuelles et anciennes. Cette fréquence tend à augmenter avec l'âge, conformément aux données de la littérature médicale, faisant état d'une relation entre ce facteur et la $\mathrm{MH}$. En effet, la maladie s'exprime généralement autour de 65 ans, rarement avant 50 ans (Morlock 1992). La répartition sexuelle des individus atteints indique une prédominance masculine de la maladie. Si ce résultat concorde avec les données épidémiologiques, la très forte fréquence de la MH chez les hommes, ainsi que le rapport des fréquences entre homme et femme $(10,4)$, sont nettement supérieurs à ceux généralement rencontrés dans les populations actuelles (Mazières, Rovensky 2000). Ces anomalies ne sont en fait imputables qu'à une partie des inhumés du cimetière. Ainsi, la fréquence masculine de la $\mathrm{MH}$ dans le cimetière ouvert et le Chœur des Dames est relativement faible et n'est que deux fois plus importante que la fréquence féminine, rapport classiquement rencontré (Mazières, Rovensky 2000). À l'opposé, une grande proportion des hommes inhumés dans la chapelle et la chapelle cémétériale est atteinte de MH. Le taux d'indétermination sexuelle ne semble pas pouvoir à lui seul expliquer cette fréquence élevée. En effet, en admettant que tous les individus de sexe indéterminé provenant des chapelles soient masculins, la fréquence de la $\mathrm{MH}$ dans ce secteur $(16,7 \%-20,8 \%)$ demeurerait largement supérieure à celle observée dans le reste du cimetière.

Si les sources textuelles ne nous permettent pas de certifier l'identité de chaque squelette, elles indiquent clairement la volonté d'individus de rang social élevé d'être inhumés dans certains lieux. Malgré un effectif relativement faible de malades, ces données constituent un avantage majeur pour discuter de la relation entre la $\mathrm{MH}$ et le statut social des individus. La fréquence élevée de la $\mathrm{MH}$ dans les chapelles pourrait, selon nous, s'expliquer de deux manières. D'une part, des individus bourgeois ou religieux pourraient avoir eu une vie moins éprouvante que le reste de la population et ainsi bénéficié d'une plus grande longévité. Ces individus «privilégiés » pourraient alors avoir une plus grande probabilité de présenter les lésions osseuses de la maladie. D'autre part, les classes aisées auront eu accès, selon toute vraisemblance, à des sources alimentaires plus abondantes que le reste de la population. Une diète trop riche pourrait être à l'origine d'un risque accru de développer la $\mathrm{MH}$. Cette deuxième hypothèse, fondée sur des données médicales faisant état d'une relation entre la $\mathrm{MH}$ et différents désordres métaboliques (Julkunen et al. 1971 ; Utsinger 1985 ; Lussier, de Medicis 1991), a déjà été discutée dans plusieurs études paléopathologiques (Rogers, Waldron 2001 ; Jankauskas 2003 ; Gleize, Goude 2005). Dans le cas présent, l'absence de différence significative dans la répartition par âge au décès des individus masculins entre les secteurs d'inhumation est en faveur de cette interprétation. Toutefois, la forte proportion d'individus d'âge indéterminé doit nous inviter à la plus grande prudence. En l'absence de données cliniques sur le statut métabolique des individus, il reste difficile de confirmer cette hypothèse (Vidal 2000) et d'éliminer le simple effet d'une différence de longévité entre groupes sociaux au sein de la population.

\section{CONCLUSIONS}

Les résultats obtenus, s'ils ne permettent pas de statuer de manière définitive sur le lien entre $\mathrm{MH}$ et régime alimentaire des individus, semblent indiquer une relation entre le statut social supposé des inhumés et l'expression de la maladie. Cette étude confirme l'intérêt d'une démarche interdisciplinaire pour la reconstitution du mode de vie des populations du passé. Elle démontre l'apport de l'analyse de la répartition spatiale des cas de MH pour discuter du statut social des individus. Cette approche semble d'autant plus pertinente pour les périodes récentes, pour lesquelles le caractère codifié du rite funéraire et la pauvreté du mobilier limitent les informations archéologiques concernant le rang social des inhumés.

\section{Remerciements}

Nous adressons nos remerciements au Service Archéologique de la ville de Beauvais, ainsi qu'à D. Castex et P. Courtaud pour nous avoir permis d'étudier cette série ostéologique. Nous tenons également à remercier P. Murail, P. Vidal et les deux relecteurs anonymes pour leur commentaires et suggestions. Enfin, nous remercions la Maison des Sciences de l'Homme d'Aquitaine (S.K.) et le Ministère de la Recherche (S.V.) pour leur soutien financier. 


\section{BIBLIOGRAPHIE}

Benjamin (M.), Mcgonagle (D.) 2001, The anatomical basis for disease localisation in seronegative spondyloarthopathy at entheses and related sites, Journal of Anatomy 199: 503-526.

Chevalier (N.) 1993, Le cimetière du couvent des Sours Grises de Beauvais de la fin du XV $V^{e}$ au XVIII ${ }^{e}$ siècle, Étude des pratiques funéraires, Mémoire de DEA, Université Bordeaux 1 (Inédit).

Crubézy (E.) 1990, Diffuse Idiopathic Skeletal Hyperostosis: Diagnosis and Importance in Paleopathology, Journal of Paleopathology 3, 2: 107-118.

Crubézy (É.), Causse (L.), Delmas (J.), Ludes (B.) 1998, Le paysan médiéval en Rouergue, Cimetière et église de Canac (Campagnac, Aveyron), Musée archéologique de Montrosier, Aveyron, $264 \mathrm{p}$.

CunHa (E.) 1993, Évolution de la maladie hyperostosique à Coimbra de l'époque médiévale à la fin du $\mathrm{XIX}^{\mathrm{e}}$ siècle, Bulletins et Mémoires de la Société d'Anthropologie de Paris, n.s., 5, 1-2 : 313-320.

FÉMOLANT (J.-M.) 1992, Beauvais Caserne Taupin « Antenne Universitaire », Bilan scientifique, Service Régional de l'Archéologie de Picardie, p. 48-50.

Forestier (J.), Rotès-Querol (J.) 1950, Hyperostose ankylosante vertébrale sénile, Revue du Rhumatisme $10: 525-534$.

Gleize (Y.), Goude (G.) 2005, Anthropologie, biochimie et alimentation, in E. Normand, C. Treffort (éds), À la table des moines charentais, Archéologie de l'alimentation monastique en Charente et Charente-Maritime, Geste éditions, La Crèche, p. 60-61.

JANKAUSKAS (R.) 2003, The Incidence of Diffuse Idiopathic Skeletal Hyperostosis and Social Status in Lithuanian Skeletal Materials, International Journal of Osteoarchaeology 13: $289-293$

Julkunen (H.), Heinonen (O.P.), Pyorala (K.) 1971, Hyperostosis of the spine in an adult population, Its relation to hyperglycaemia and obesity, Annals of the Rheumatic Diseases 30: 605-612.

LAGIER (R.) 1987, Approche globale du concept d'arthrose par l'anatomopathologie, in L. Simon, G. Loyau (éds), L'arthrose : perspectives et réalités, Masson, Paris, p. 185-197.
Lussier (A.), Medicis (R. de) 1991, Maladie hyperostosique : renouveau étiopathogénique et clinique, in L. Simon, C. Hérisson, J. Rodineau (éds), Pathologie des insertions et enthésopathies, Masson, Paris, p. 84-91.

MAZIÈRes (B.), Rovensky (J.) 2000, Non-inflammatory enthesopathies of the spine: a diagnostic approach, Baillière's Clinical Rheumatology 14: 201-217.

MorLocK (G.) 1992, Hyperostose vertébrale ankylosante et vieillissement rachidien, in L. Simon, C. Hérisson, B. Biot (éds), Le rachis vieillissant, Masson, Paris, p. 106-114.

Murail (P.), BruzeK (J.), Braga (J.) 1999, A New Approach to Sexual Diagnosis in Past Populations, Practical Adjustments from Van Vark's Procedure, International Journal of Osteoarchaeology 9: 39-53.

Murail (P.), BruzeK (J.), HouËt (F.), Cunha (E.) 2005, DSP: A Tool for Probabilistic Sex Diagnosis Using Worldwide Variability in Hip-Bone Measurements, Bulletins et Mémoires de la Société d'Anthropologie de Paris, n.s., 17, 3-4: 167-176.

Owings-WebB (P.A.), Suchey (J.M.) 1985, Epiphyseal union of the anterior iliac crest and the medial clavicle in a modern multiracial sample of American males and females, American Journal of Physical Anthropology 68: 457-466.

RESNICK (D.) 2002, Diffuse idiopathic skeletal hyperostosis, in D. Resnick (ed.), Diagnosis of bone and joint disorders, 4th edition, Saunders, Philadelphia, p. 1271-1381.

Resnick (D.), Shaul (S.R.), Robins (J.M.) 1975, Diffuse idiopathic skeletal hyperostosis (DISH): Forestier's disease with extraspinal manifestations, Diagnostic Radiology 119: $559-568$.

Rogers (J.), WALDRON (T.) 1995, A Field Guide to Joint Disease in Archaeology, John Wiley and Sons Ltd, Chichester, $128 \mathrm{p}$.

Rogers (J.), WALDRON (T.) 2001, DISH and the Monastic Way of Life, International Journal of Osteoarchaeology 11: $357-365$

SchmitT (A.) 2005, Une nouvelle méthode pour estimer l'âge au décès des adultes à partir de la surface sacro-pelvienne iliaque, Bulletins et Mémoires de la Société d'Anthropologie de Paris, n.s., 17, 1-2 : 89-101. 
UtSINGER (P.D.) 1985, Diffuse Idiopathic Skeletal Hyperostosis, Clinics of Rheumatic Diseases 11: 325-329.

Van de Berg (B.), Lecouvert (F.), Maldague (B.), MALGHEM (J.) 2003, Modifications osseuses péri-discales et péri-vertébrales, in H. Bard, A. Cotten, J. Rodineau,
G. Saillant, J-J. Railhac (éds), Tendons et enthèses, Sauramps Médical, Montpellier, p. 29-36.

VIDAL (P.) 2000, Approche paléo-épidémiologique de la maladie hyperostosique, Revue $d u$ Rhumatisme 67 : 214-218. 